\title{
PHOTOMETRIC OBSERVATIONS OF THE CENTRAL STARS OF PLANETARY NEBULAE
}

\author{
William Liller and Cheng-Yuan ShaO \\ (Harvard College Observatory, Cambridge, Mass., U.S.A.)
}

Currently at Harvard, we are engaged in a program for measuring multi-color magnitudes of the central stars of planetary nebulae. We plan to determine magnitudes of all favourable planetaries having central stars brighter than 16th photographic magnitude. The results, when completed, will give us improved information on (1) the amount of interstellar extinction for the individual objects, (2) the luminosities and colors of the central stars, (3) interstellar reddening corrections for spectrophotometric studies, and (4) the existence of variability among the central stars.

The nebular stars are measured both photoelectrically and photographically through special filters (with effective wavelengths close to those of the $U, B, V$ system) to exclude the stronger nebular radiations, and also with standard $U, B, V$ filters. While our observations are made primarily with the $155-\mathrm{cm}$ reflector at Harvard's Agassiz Station, we also plan to use telescopes at the Kitt Peak National Observatory and at Cerro Tololo in Chile. During the 1966-67 season, we obtained data for the central stars of more than 30 planetaries, mostly NGC objects. At present, we have under observation about an equal number of stars selected from the Perek-Kohoutek Catalogue (1967).

In addition to determining the magnitudes of the nebular nuclei, we also measure some nearby stars around each nebula to check any light variation of the nucleus and to estimate the amount of interstellar reddening and extinction in the direction of the nebula. We have scheduled extensive observations of some central stars of suspected or known variability. This report summarizes some preliminary results of our program.

Table 1 lists the stars for which we have photoelectric data. The column headings $V, B-V$ and $U-B$ have their conventional meanings. The letter $W$ represents the yellow observations made with a Wratten-15 filter (transmission $\lambda 5100$ and redwards), which is used to eliminate the strong [OIII] emission from the nebulae. The $W$ magnitudes and the $B-W$ colors, transformed to the $U, B, V$ system, are given in parenthesis.

Because of the high surface brightness of most NGC objects, it is often difficult to separate entirely the central star from the surrounding nebulosity of complex structure and non-uniform intensity distribution. We always attempt to observe with the smallest diaphragms ( 8 " on the Newtonian and 3 " on the Cassegrain photometers) to

Osterbrock and O'Dell leds.). Planetary Nebulae, 320 323. ' I.A.U. 
Table 1

Preliminary magnitudes and colors of the central stars of planetary nebulae

\begin{tabular}{|c|c|c|c|}
\hline Nebula & $V($ or $W)$ & $B-V($ or $B-W)$ & $U-B$ \\
\hline 246 & 11.95 & -0.37 & $-1 \cdot 20$ \\
\hline 1501 & $(14 \cdot 2)$ & $(-0 \cdot 2)$ & -0.7 \\
\hline 1514 & $9 \cdot 42$ & +0.55 & -0.06 \\
\hline 1418 & (9.7) & -0.2 & -1.0 \\
\hline 2022 & $(14 \cdot 9)$ & +0.6 & -0.6 \\
\hline 2392 & $(10.4)$ & +0.4 & -0.7 \\
\hline A 30 & $14 \cdot 36$ & -0.06 & -1.02 \\
\hline 4361 & $12 \cdot 9$ & -0.3 & $-1 \cdot 3$ \\
\hline A 36 & $11 \cdot 43$ & -0.32 & $-1 \cdot 22$ \\
\hline 6058 & $(13 \cdot 7)$ & -0.3 & -1.0 \\
\hline 6543 & $(10 \cdot 8)$ & +0.6 & -0.5 \\
\hline 6720 & 14.7 & -0.4 & $-1 \cdot 3$ \\
\hline M 1-67 & 11.05 & +1.07 & -0.09 \\
\hline 6804 & (13.9) & +0.6 & -0.7 \\
\hline$+30^{\circ} 3639$ & $10 \cdot 10$ & -0.06 & -0.71 \\
\hline 6853 & 13.82 & -0.29 & $-1 \cdot 16$ \\
\hline 6857 & $(13 \cdot 30)$ & +1.04 & -0.15 \\
\hline 7094 & 13.60 & -0.23 & -1.20 \\
\hline 7293 & 13.50 & -0.34 & -1.30 \\
\hline
\end{tabular}

Remarks: 246,1514 , A 30 and A 36 - Nebula faint, contamination negligible. $6720,6857,7094$ and 7293 - Ring excluded. $+30^{\circ} 3639$ - Nebular disk included. 6853 - Corrected for nebula.

exclude the background nebula, but sky conditions do not always make it possible. The standard $U, B, V$ filters all transmit a number of bright nebular emissions. The $B$ and $V$ filters are 30 and $40 \%$ transparent at the wavelengths of the very strong green [OIII] lines; the [OII] pair at $\lambda 3727$ can often seriously affect the $U$ measurements, although the greater relative intensity of the central star tends to minimize this influence. The $W$ measurements, which are least affected by nebular emissions, best represent the yellow magnitudes of the stars. Therefore, except for a few objects noted in the remarks of the table, the listed $B-W$ values are too small while the $B-V$ and $U-B$ colors are too large. We are now experimenting with two blue filters, one (glass) which eliminates the green nebular lines and the other (interference) which transmits only the spectrum between $\mathrm{H} \gamma$ and $\mathrm{H} \delta$ lines.

For the objects not suitable for photoelectric measurements, the data have been supplemented by photographic observations with 'anti-nebula' filters. On moderately exposed plates the nebular material can be seen more easily than at the photometer eyepiece through diaphragms. Furthermore, it is possible to use defining apertures (such as an iris photometer provides) that are no larger than the star image itself. For this work, we have established photoelectric secondary standards in the immediate neighbourhood of each central star. 
A few objects of special interest have received particular attention and we discuss them in turn.

$N G C$ 1514: Because the nebula of this object is much fainter than its central star, the nebula did not appreciably contaminate the photoelectric observations of the star. The colors we observed for the $\operatorname{star}(B-V=+0 \cdot 55, U-B=-0.06)$ and for other stars in its vicinity indicate a $B-V$ color excess $\sim 0$. 5 , in agreement with the results of $\mathrm{Ko}$ houtek (1966), who suggests that the central star is a reddened A0 star with appreciable ultraviolet excess attributed to an invisible hotter companion.

If the true nucleus of NGC 1514 is actually a double system with an O-type star fainter than its apparent $\mathrm{A} 0$ nucleus, the presence of the hotter component might be detectable by observations at very short wavelengths. In order to do so, we observed the star with a photoelectric spectral scanner at Agassiz Station on October 13, 1966. Two scans (Figure 1) from $3100 \AA$ to $5400 \AA$ showed that the central star is indeed a reddened star of the expected spectral type with detectable excess ultraviolet radiation.

Kohoutek 1-2: The nucleus of this planetary was found (Kohoutek, 1964) to be

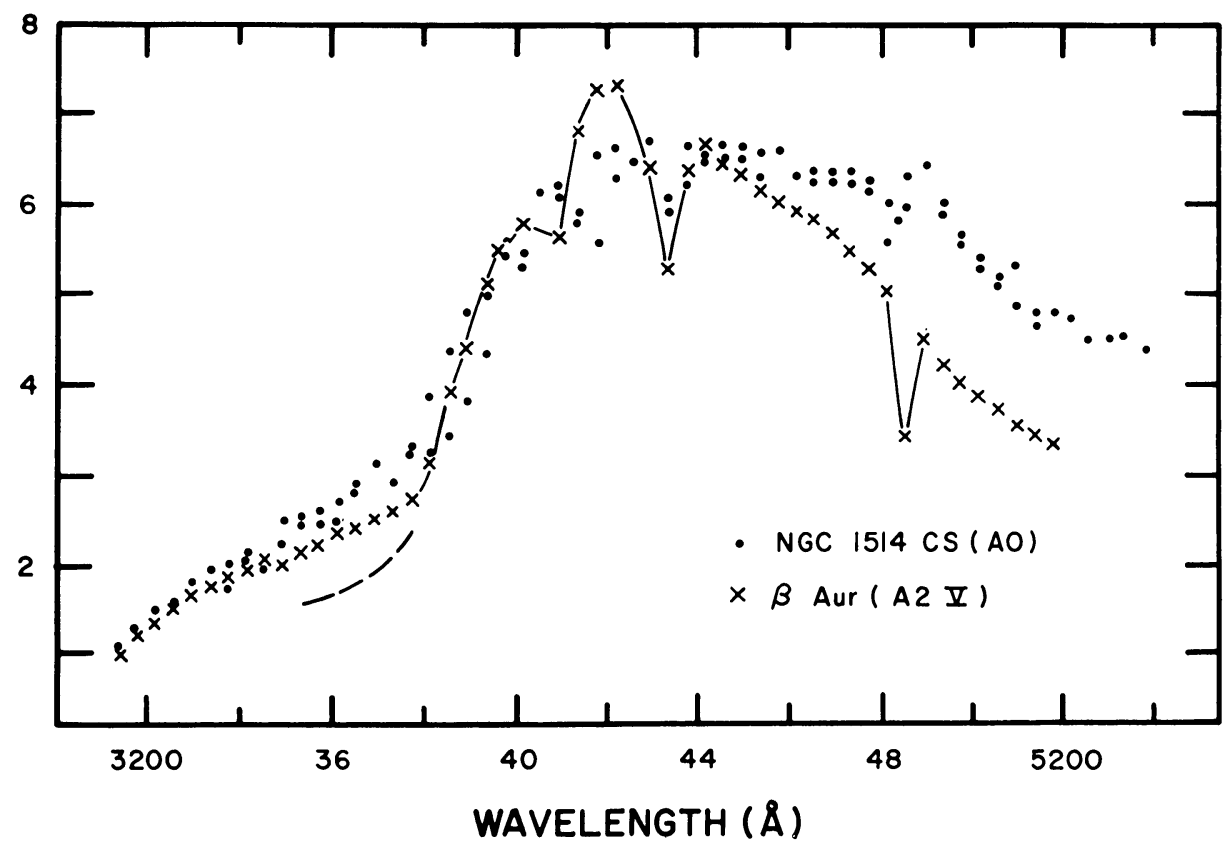

FIG. 1. Spectrophotometric scans of the central star of NGC 1514. The two scans of the central star from $3100 \AA$ to $5400 A, \Delta \lambda=50 A$, are plotted together with the single scan of $\beta$ Aur. Each dot or cross represents one observation. At the red end of the spectrum, the intensity of the reddened nucleus is higher than that of $\beta$ Aur. The spectrum of an AO star reddened by $\sim 0^{\mathrm{m}} .5$ in $B-V$ is estimated to fall along the dashed line at the UV end, whereas the observed central-star continuum is higher (color bluer), confirming the existence of an UV excess. The ordinate is relative intensity. 
conspicuously variable on the overlap portion of two Palomar Sky Survey prints for two successive nights. From a search of the Harvard photographic plate collection, we confirmed the reported variability of the star. All available data appear in Figure 2, which shows that the star's photographic magnitude varies from $15 \cdot 7$ to at least $17 \cdot 4$. While the star spends most of its time at an intermediate magnitude (16.6 average), brightness changes of 1.5 are possible in about 1 day. These facts seem to suggest irregular fluctuations. We plan to make more observations from Kitt Peak in October and November 1967 to further the study of its interesting light variation.

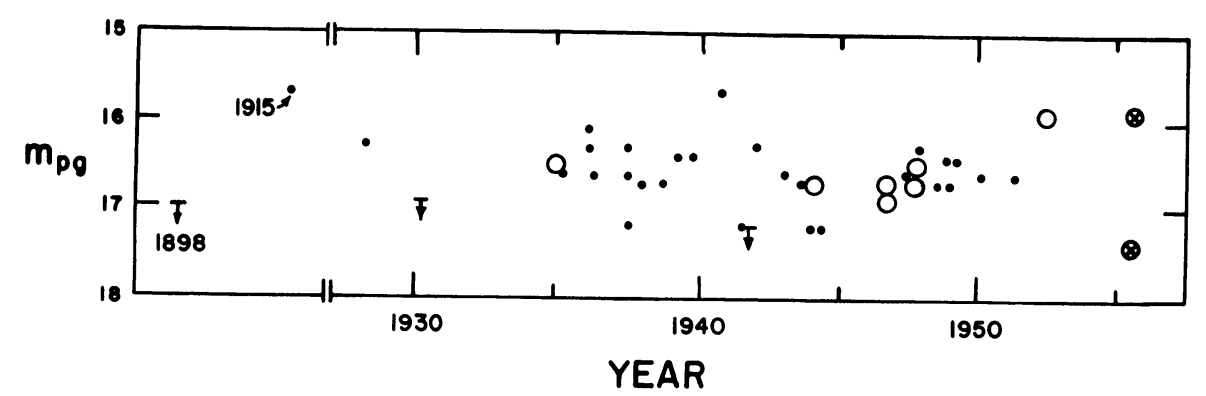

FIG. 2. Photographic light variation of the central star of Kohoutek 1-2. Circles represent observations with an accuracy of $\pm 0^{\mathrm{m}} .1$, and dots $\pm 0^{\mathrm{m}} .3$. The two crossed circles indicate magnitudes from the Palomar Sky Survey prints. Arrows show when the star was fainter than the plate limit.

Abell 36: This nebula possesses the brightest central star $(V=11 \cdot 5)$ in Abell's (1966) list of objects. The colors we measured agree well with those found by him, but the $V$ magnitude differs by $0 \cdot 1$ magnitude. Because he suggested the possible variability of this star, we examined some 200 Harvard plates taken between 1931 and 1952 . No light variation $( \pm 0.2)$ was found from our visual estimates.

\section{Acknowledgements}

We thank Dr. Martha Liller for her help and the National Science Foundation for its support.

\section{References}

Abell, G.O. (1966) Astrophys. J., 144, 259.

Kohoutek, L. (1964) Bull. astr. Inst. Csl., 15, 161.

Kohoutek, L. (1966) Bull. astr. Inst. Csl., 18, 103.

Perek, L., Kohoutek, L. (1967) Catalogue of Galactic Planetary Nebulae, Academia, Prague. 